\title{
Hemodynamics of dexamethasone-induced hypertension in the rat
}

\author{
Sharon Leng Hong Ong, Yi Zhang, Matthew Sutton and Judith Ann Whitworth
}

Although dexamethasone (DEX) is known to cause hypertension in humans and in animals, the hemodynamic characteristics of DEX-induced hypertension (DEX-HT) in the rat remain unclear. This study evaluated central and regional hemodynamics, and the role of total peripheral resistance (TPR) using a vasodilator minoxidil. Rats were divided into four groups, namely saline $(n=20)$, DEX $(n=21)$, minoxidil+saline $(n=10)$ and minoxidil+DEX $(n=10)$. Tail-cuff systolic blood pressure was recorded every second day. After 10-14 days of treatment, central (saline: $n=9$, DEX: $n=10$ ) and regional (saline: $n=11$, DEX: $n=11$ ) hemodynamic parameters were measured. Central hemodynamic data were also obtained from minoxidil-treated rats. DEX increased blood pressure $(P<0.0005)$ in association with an increase in TPR $(P<0.05)$. However, individual assessments of renal, mesenteric and hindquarter circulations did not detect any significant increase in resistance in these beds. Minoxidil increased cardiac output $\left(P^{\prime}<0.01\right)$ and cardiac index $\left(P^{\prime}<0.005\right)$ as well as decreased TPR $(P<0.05)$ without affecting DEX-HT. DEX prevented weight gain and decreased thymus weight. The increase in TPR in DEX-HT in rats was not simply explained by isolated alterations to resistance in the renal, mesenteric or hindquarter circulations. Minoxidil effectively prevented the increase in TPR but not the increase in blood pressure, suggesting that an increase in TPR is not essential for DEX-induced blood pressure increase.

Hypertension Research (2009) 32, 889-894; doi:10.1038/hr.2009.118; published online 31 July 2009

Keywords: dexamethasone; hemodynamics; minoxidil; total peripheral resistance

\section{INTRODUCTION}

Synthetic glucocorticoid dexamethasone (DEX) is commonly used in clinical settings for the treatment of various medical conditions. It is used in adrenal insufficiency as a glucocorticoid hormone replacement treatment, in multiple myeloma together with other chemotherapeutic agents, to decrease cerebral edema due to intracranial pathology and as an anti-emetic in patients with cancer. When used chronically at a supra-physiological dose, DEX results in hypertension. The underlying mechanism for DEX-induced hypertension (DEX-HT) remains unclear. We have recently reviewed postulated mechanisms of DEXHT. ${ }^{1}$ There is evidence against plasma volume expansion ${ }^{2}$ or sympathetic nerve activation ${ }^{3}$ as key mechanisms of DEX-HT in humans. The roles of endogenous vasoconstrictors and vasodilators in DEX-HT are variable, with possible associations with nitric oxide, prostanoids, angiotensin II, arginine vasopressin, endothelins, catecholamines, neuropeptide $\mathrm{Y}$ and atrial natriuretic peptide. ${ }^{1}$ Studies on DEX-HT in rodents have indicated that nitric oxide deficiency ${ }^{4}$ and oxidative stress ${ }^{5}$ are implicated in this form of hypertension. Nevertheless, the hemodynamic profile has not been fully evaluated in this model.

The hemodynamics of DEX-HT has been evaluated in both humans $^{6}$ and dogs. ${ }^{7}$ DEX-HT in humans ( $3 \mathrm{mg}$ per day) was associated with increased total peripheral resistance (TPR) without any change in cardiac output (CO). ${ }^{6}$ Although these are useful data depicting the hemodynamic patterns of DEX-HT, little is known about the regional flow patterns contributing to increased TPR. In dogs, DEX-HT $\left(0.5 \mathrm{mg} \mathrm{kg}^{-1}\right.$ per day) was found to be associated with increased TPR and decreased $\mathrm{CO}^{7}$ Whether these changes represent features necessary for the development of DEX-HT is not known. In that study, only CO, TPR and renal blood flow were evaluated, leaving other hemodynamic parameters unexamined. Furthermore, the relevance of the dog model of DEX-HT to that in humans remains unclear. For examining the mechanisms of glucocorticoid-induced hypertension, the rat may be more applicable to the human situation. 8,9

In this study, we evaluated the hemodynamic profiles of DEX-HT using ultrasonic transit-time flowmetry. This method has been validated for the measurement of $\mathrm{CO}$ and regional blood flow in rats. ${ }^{10}$ As increased TPR was observed in DEX-HT in rats, we proceeded with a second series of experiments to examine the role of TPR in DEX-HT in rats by using minoxidil, a pyrimidine derivative used in the treatment of hypertension. It is a potent vasodilator that also increases $\mathrm{CO}$ and heart rate (HR). It is generally not used as a single agent in the treatment of hypertension, as it can cause reactive fluid retention and an increase in left ventricular mass. In this study, minoxidil at $15 \mathrm{mg} \mathrm{kg}^{-1}$ per day was used, as it has been shown to be effective in reducing TPR in rats. ${ }^{11}$ 


\section{METHODS}

\section{Experimental animals}

This study was approved by the Animal Experimentation Ethics Committee of the Australian National University (Protocol no. J.HB.22.06). A total of 61 male Sprague-Dawley rats (body weight 200-300 g) were housed in plastic cages with a controlled temperature of between $21-23^{\circ} \mathrm{C}$ and a 12 -h light-dark cycle. The rats had free access to standard rat chow and tap water. They were acclimatized for 7 days to their surroundings, handling and tail-cuff sphygmomanometer before commencing the experiment.

\section{Treatment protocols}

We conducted two sets of experiments to evaluate the hemodynamics of DEXHT. The first set evaluated the hemodynamic profiles of DEX-HT and the second examined the role of TPR in DEX-HT.

In the first experiment, central and regional hemodynamic profiles of DEXHT were examined. A total of 19 rats (sham, $n=9$ and DEX treatment, $n=10$ ) were used in the central hemodynamics study and 22 rats (sham, $n=11$ and DEX treatment, $n=11$ ) in the regional hemodynamics study. These rats received saline by gavage $(0.1 \mathrm{ml}$ per $100 \mathrm{~g}$ body weight per day, from day 1 to 16$)$ and subcutaneous (s.c.) saline ( $0.2 \mathrm{ml}$ per rat per day) or DEX injections (20 $\mu \mathrm{g}$ per rat per day, s.c., David Bull Laboratories, Mulgrave, Victoria, Australia) from day 5 to 16

In the second experiment, all rats $(n=20)$ received minoxidil $\left(15 \mathrm{mg} \mathrm{kg}^{-1}\right.$ per day, Sigma-Aldrich, St Louis, MO, USA) daily by gavage between 1500 and 1600 hours for 16 days (days 1-16). From day 5 to 16 , they were also administered either DEX $(n=10)$ or sham $(n=10)$ s.c. injections daily between 1500 and 1600 hours. The data from this experiment were compared with those of the control rats (sham control, $n=9$ and DEX control, $n=10$ ) that underwent central hemodynamic experiments in Experiment 1.

\section{Blood pressure and body weight measurements}

Noninvasive systolic blood pressure (SBP) was measured in conscious rats between 0900 and 1100 hours on alternate days using a tail-cuff sphygmomanometer (Narco Biosystems, Houston, TX, USA). Several readings were recorded and the mean of four median readings, among which the difference was not $>10 \mathrm{~mm} \mathrm{Hg}$, was accepted. Body weights were measured on alternate days after tail-cuff SBP measurements.

Direct blood pressure measurements were recorded under pentobarbitone anesthesia $\left(60 \mathrm{mg} \mathrm{kg}^{-1}\right.$, intraperitoneally (i.p.), Merial Australia, Sydney, New South Wales, Australia) on the last day of experiment (day 16). Mean arterial pressure (MAP), SBP and diastolic blood pressure (DBP), as well as HR were measured using a catheter tip pressure transducer (Mikro-Tip SPR-320; Millar Instruments, Houston, TX, USA) that was inserted into the left carotid artery and advanced into the level of the aortic arch.

\section{Surgical approaches for $\mathrm{CO}$ and regional flow measurements} Cardiac output was measured using a Transonic 3SS perivascular probe (Transonic System, Ithaca, NY, USA), a Transonic T106 small animal flowmeter (Transonic System) and a PowerLab data acquisition system (ADInstruments, Sydney, New South Wales, Australia). Rats were anesthetized on a heating pad in a dorsally recumbent position, mechanically ventilated (Harvard Apparatus, South Natick, MA, USA) and administered intravenous saline ( $1 \mathrm{ml}$ per $\mathrm{h}$ ) through a tail vein intravenous cannula. Bilateral thoracotomy was performed to expose the heart and ascending aorta. The ascending aorta was isolated and the probe was positioned around the ascending aorta. Acoustic gel (SurgiLube; W Fougera, Melville, NY, USA) was deposited into the acoustic window of the probe adjacent to the artery while replacing the air space. After $20 \mathrm{~min}$ of stabilization, $\mathrm{CO}$ measurements were recorded at 1 -min intervals for $10 \mathrm{~min}$ and averaged.

Regional blood flow readings were measured using a 1RB perivascular probe (Transonic System) and the same flowmeter and data acquisition system. Midline laparotomy was performed in anesthetized rats. The left renal artery, superior mesenteric artery and left common iliac artery were carefully located and isolated. The $1 \mathrm{RB}$ perivascular probe was then placed around these arteries sequentially for blood flow measurements. All measurements were recorded at 1-min intervals after at least $20 \mathrm{~min}$ of stabilization and averaged.

\section{Calculation of other hemodynamic parameters}

- Cardiac index $(\mathrm{CI})=\mathrm{CO} / 100 \mathrm{~g}$ body weight

- Stroke volume $(\mathrm{SV})=\mathrm{CO} / \mathrm{HR}$

- Stroke index $(\mathrm{SI})=\mathrm{CI} / \mathrm{HR}$

- $\quad$ Total peripheral resistance $(\mathrm{TPR})=\mathrm{MAP} / \mathrm{CO}$

- Regional vascular resistance $=\mathrm{MAP} /$ regional blood flow

\section{Hematocrit estimation}

After the hemodynamic experiments, the animals were killed by exsanguination. Blood was collected into heparinized capillary tubes and centrifuged. Hematocrit was measured using a micro-hematocrit reader (Hawksley and Sons, Lancing, UK). Data were expressed as percentage of red blood cells per total volume of blood.

\section{Thymus weight measurement}

Thymus wet weight per $100 \mathrm{~g}$ body weight was obtained as a marker of glucocorticoid activity.

\section{Statistical analysis}

Statistical analysis was carried out using SPSS software (version 15.0, SPSS, Chicago, IL, USA). Results were expressed as mean \pm s.e.m. Results were analyzed by unpaired $t$-test or repeated-measures analysis of variance with Greenhouse-Geisser adjustment for multisample asphericity. The Ryan-Holm step down Bonferroni procedure was applied to raw $P$-values to control the family-wise type 1 error. $P<0.05$ or Bonferroni-adjusted $P$-value $\left(P^{\prime}\right) \leqslant 0.05$ was considered significant.

\section{RESULTS}

\section{Hemodynamic profiles of DEX-HT}

Tail-cuff SBP. In the first experiment, sham injections with sterile saline did not alter SBP $(126 \pm 3 \mathrm{~mm} \mathrm{Hg}$ on day $5,124 \pm 2 \mathrm{~mm} \mathrm{Hg}$ on day $16, n=21)$. DEX significantly increased SBP from $123 \pm 2 \mathrm{~mm} \mathrm{Hg}$ on day 5 to $142 \pm 2 \mathrm{~mm} \mathrm{Hg}$ on day $16 \quad(n=20, P<0.0005)$. In comparison with saline-treated rats $(n=20)$, SBP in the DEX-treated group $(n=21)$ was significantly higher $(P<0.0005)$ (Figure 1a).

Central hemodynamics. Dexamethasone-treated rats $(n=10)$ had significantly higher MAP $(P<0.0005)$, SBP and DBP $(P<0.0005)$, as well as TPR $\left(P^{\prime}<0.05\right)$ levels in comparison with the sham treatment group $(n=9)$. There was no significant difference in HR, CO, CI, SV and SI between the DEX $(n=9)$ and sham $(n=10)$ treatment groups (Table 1).

Regional hemodynamics. In comparison with sham treatment $(n=11)$, DEX treatment $(n=11)$ increased MAP $(P<0.05)$, SBP $(P<0.005)$ and DBP $(P<0.05)$, but did not significantly change renal, mesenteric or common iliac blood flow or vascular resistance measurements (Table 2).

\section{Role of TPR in DEX-HT}

Tail-cuff SBP. Dexamethasone treatment increased tail-cuff SBP from $125 \pm 4$ to $140 \pm 3 \mathrm{~mm} \mathrm{Hg}$ (days $5-16, P<0.005, n=9$ ), whereas sham injections with saline did not alter SBP (day 5: $128 \pm 4 \mathrm{~mm} \mathrm{Hg}$, day 16: $120 \pm 4 \mathrm{~mm} \mathrm{Hg}, n=10$ ). Tail-cuff SBP in the DEX-treated group $(n=10)$ was significantly higher than that in the sham treatment group $(P<0.005, n=9)$. The increase in tail-cuff SBP in rats treated with minoxidil $+\mathrm{DEX}$ was not significant $(P=0.086, n=10)$. Minoxidil treatment did not alter SBP in saline-treated rats (Figure $1 \mathrm{~b}$ ).

Direct blood pressure measurements. Both direct SBP and DBP measurements obtained on day 16 were significantly higher in DEXtreated rats than in saline-treated (control) rats. Rats that were on 

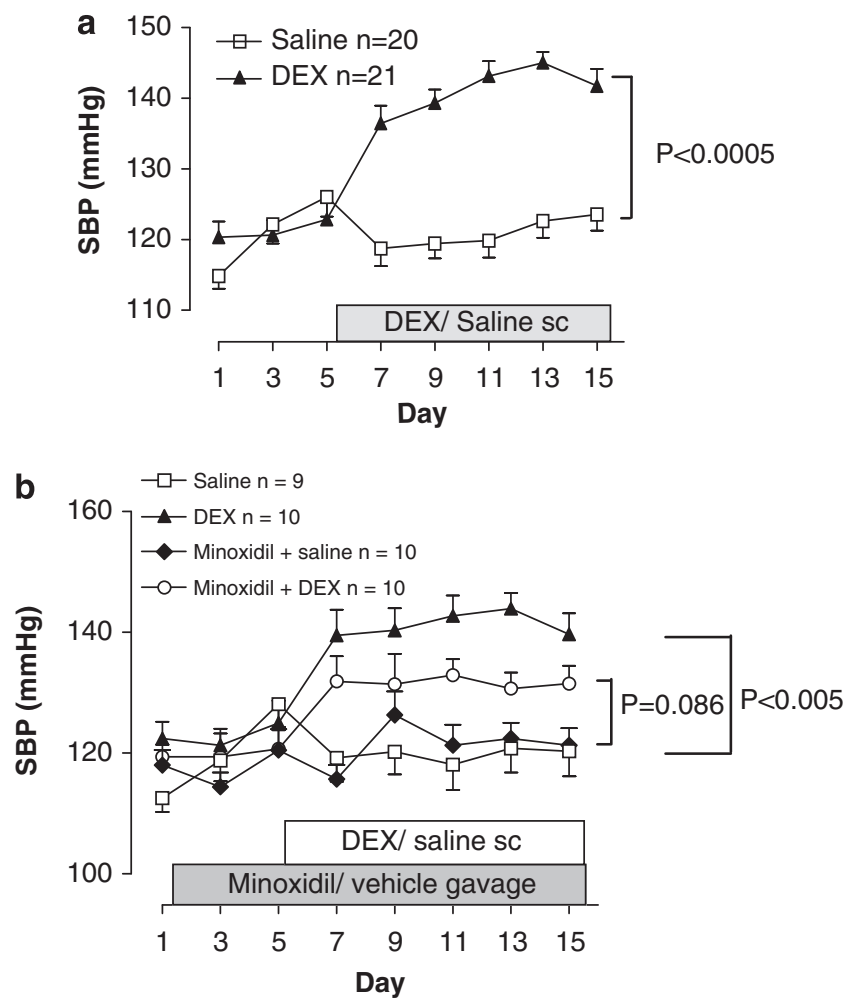

Figure 1 Tail-cuff systolic blood pressure. (a) Experiment 1: ( $\square$ ) Saline

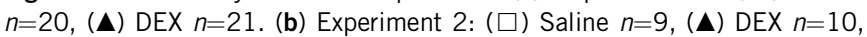
$(\diamond)$ Minoxidil+saline $n=10,(O)$ Minoxidil+DEX $n=10$. DEX, dexamethasone; SBP, systolic blood pressure.

minoxidil+DEX treatments had a significantly lower SBP than did those on DEX treatment alone $\left(P^{\prime}<0.05\right)$. However, there was no significant difference in direct DBP between DEX- and minoxidil+DEX-treated rats. Minoxidil treatment significantly increased direct DBP $\left(P^{\prime}<0.05\right)$ but not direct SBP or MAP in rats that received saline injections.

Mean arterial pressure was significantly higher in rats on DEX than in those on saline $\left(P^{\prime}<0.01\right)$. This increase was not prevented by minoxidil. Minoxidil did not alter MAP in saline-treated rats (Table 1).

Heart rate. Heart rate was not significantly altered by DEX or minoxidil treatments (Table 1 ).

Stroke volume and stroke index. There was no significant difference in SV and SI between the DEX-treated and sham groups. In comparison with the sham group, rats on minoxidil+sham treatments had a significantly higher SV $\left(P^{\prime}<0.005\right)$ and SI $\left(P^{\prime}<0.05\right)$. Similarly, minoxidil+DEX treatments resulted in a significantly higher SV $\left(P^{\prime}<0.005\right)$ and SI $\left(P^{\prime}<0.005\right)$ compared with DEX treatment alone (Table 1).

Cardiac output and cardiac index. Minoxidil+sham treatments significantly increased CO $\left(P^{\prime}<0.01\right)$ and CI $\left(P^{\prime}<0.005\right)$ in comparison with the sham group. Similarly, minoxidil+DEX-treated rats had a significantly higher CO $\left(P^{\prime}<0.01\right)$ and $\mathrm{CI}\left(P^{\prime}<0.005\right)$ compared with DEX-treated rats (Table 1 ).

Total peripheral resistance. Total peripheral resistance was significantly increased in the DEX-treated group compared with the sham

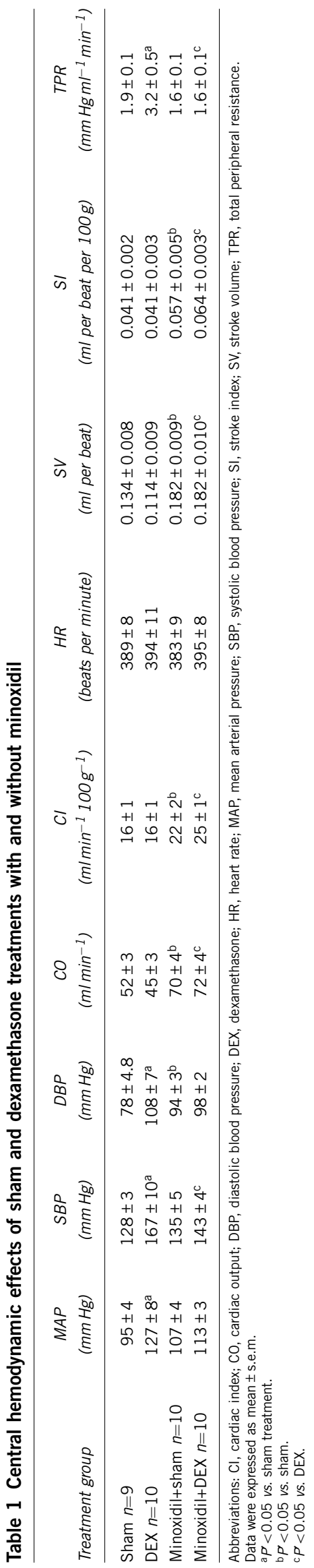




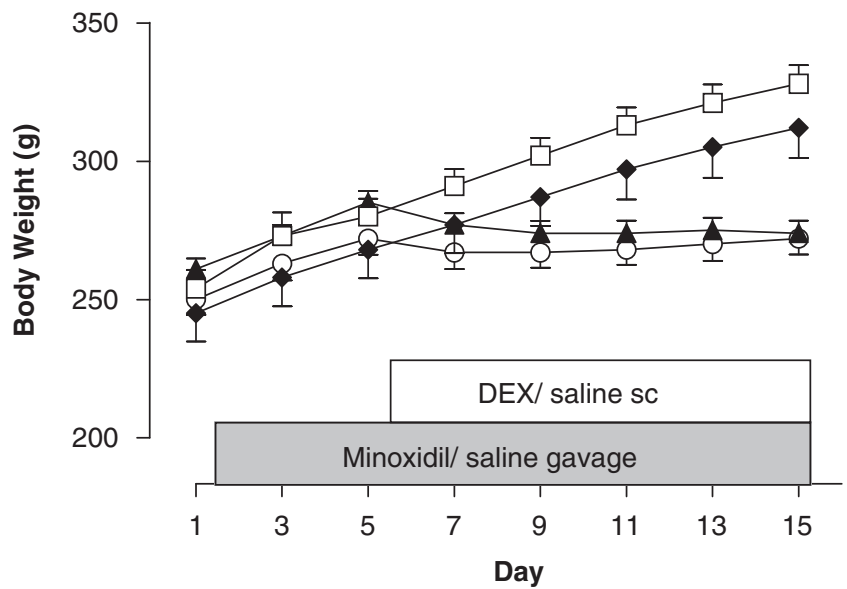

Figure 2 Body weight. ( $\square$ ) Saline $n=20$, ( $\mathbf{\Delta})$ DEX $n=21$, ( $\bullet$ ) Minoxidil+ saline $n=10,(\bigcirc)$ Minoxidil+DEX $n=10$. DEX, dexamethasone.

Table 3 Thymus weight and hematocrit

\begin{tabular}{lcc}
\hline Groups & Thymus (mg per 100g body weight) & Hematocrit (\%) \\
\hline Sham $(n=20)$ & $163 \pm 9$ & $56.4 \pm 0.6$ \\
DEX $(n=21)$ & $53 \pm 5^{\mathrm{a}}$ & $57.7 \pm 0.8$ \\
Minoxidil+sham $(n=10)$ & $172 \pm 10$ & $51.9 \pm 0.6^{\mathrm{b}}$ \\
Minoxidil+DEX $(n=10)$ & $60 \pm 4^{\mathrm{c}}$ & $54.5 \pm 1.6$ \\
\hline Abbreviation: DEX, dexamethasone. & & \\
Data were expressed as mean \pm s.e.m. & & \\
a $P<0.0005$ vs. sham treatment. & \\
b $P<0.005$ vs. sham. & & \\
&
\end{tabular}

treatment group $\left(P^{\prime}<0.05\right)$ and was significantly lower in the minoxidil+DEX-treated group in comparison with the DEX-treated group $\left(P^{\prime}<0.05\right)$ (Table 1$)$.

\section{Body weight}

There was an initial decrease in body weight from $285 \pm 4 \mathrm{~g}$ on day 5 , after the initiation of DEX treatment, to $277 \pm 4 \mathrm{~g}$ on day 7 $(P<0.0005)$. Subsequently, body weight remained stable at that level throughout the entire treatment period $(n=21)$. Conversely, rats on sham treatment gained weight progressively over the entire experimental period from $280 \pm 7 \mathrm{~g}$ on day 5 to $328 \pm 7 \mathrm{~g}$ on day $16(n=20$, $P<0.0005)$. Minoxidil did not alter DEX-induced body weight changes (Figure 2).

\section{Hematocrit}

There was no significant difference in hematocrit between saline and DEX-treated rats. Minoxidil+sham treatments produced a significant decrease in hematocrit compared with sham treatment alone. This decrease was not seen in minoxidil+DEX-treated rats (Table 3).

\section{Thymus weight}

Dexamethasone treatment, regardless of the presence of minoxidil treatment, decreased thymus weight (Table 3 ).

\section{DISCUSSION}

Dexamethasone increased blood pressure and TPR but did not alter CO, CI, SV or SI in the rat. However, the evaluation of renal, mesenteric and hindquarter circulations that were performed sepa- 
rately did not identify any significant increase in resistance in these beds. Minoxidil treatment significantly decreased TPR and increased CO in DEX-hypertensive rats but did not prevent hypertension (measured using the tail-cuff method).

The increase in TPR observed in this study is consistent with the results obtained from DEX-hypertensive humans ${ }^{6}$ and dogs. ${ }^{12}$ It is unlikely that alterations in blood viscosity, which could in turn alter vascular resistance, contribute to the increase in TPR in DEX-HT. In this study, DEX-HT was not associated with any change in hematocrit. This finding was different from that reported by $\mathrm{Hu}$ et al. ${ }^{5}$ and Tonolo et al. ${ }^{13}$ showing that DEX at lower doses $\left(20 \mu \mathrm{g} \mathrm{kg}^{-1}\right.$ per day, which is approximately $5-6 \mu \mathrm{g}$ per rat per day and $2 \mu \mathrm{g}$ per rat per day) resulted in a small but significant increase in hematocrit in rats. It is possible that the small increase in hematocrit due to DEX treatment in this study was masked by interventions during surgical procedures, such as intravenous saline administration. It is also not clear whether differences in the dose of DEX had an effect in the observed discrepancy. The effect of DEX on hematocrit and plasma osmolality was different in other species. In humans, DEX-HT was not associated with increased hematocrit. ${ }^{14}$ DEX-HT in dogs was not accompanied by a significant alteration in plasma osmolality. ${ }^{7}$

Another explanation for the increase in TPR is DEX-induced changes to vascular tone. There is increasing evidence linking deficiency in vasorelaxant nitric oxide to the pathogenesis of DEX-HT. Plasma nitrate/nitrite, a marker of nitric oxide synthesis, was significantly reduced in DEX-hypertensive humans ${ }^{14}$ and in rodents. ${ }^{4,15}$ There are also reports implicating DEX in potentiating vascular pressor responsiveness to noradrenaline and/or angiotensin II in humans, ${ }^{6}$ rats $^{16}$ and dogs. ${ }^{7}$

Despite detecting an increase in TPR, we did not observe any significant differences in blood flow or resistance in individual renal, mesenteric or hindquarter vascular beds in this study. It is possible that the observed increase in TPR may reflect cumulative changes from a number of peripheral vascular beds, including those evaluated in this study. In addition, these changes could also be a consequence of alterations in a site or sites other than the renal, mesenteric or hindquarter vascular system. Furthermore, this technique may not be sensitive enough to detect a change in the different vascular beds separately. DEX administration at a significantly higher dose $\left(3 \mathrm{mg} \mathrm{kg}^{-1}\right.$ or $\sim 900 \mu \mathrm{g}$ per rat per day, continuous infusion over $24 \mathrm{~h}$ ) in conscious rats resulted in increased MAP and decreased renal and mesenteric blood flows. ${ }^{17}$ These differences could be due to various factors, such as DEX dose differences, duration of the study and anesthesia. Data obtained from studies using a high DEX dose need to be interpreted with caution. Volume depletion through diuresis $^{18}$ due to high-dose DEX may confound hemodynamic assessments of DEX-HT.

Oral minoxidil at $15 \mathrm{mg} \mathrm{kg}^{-1}$ per day has been previously shown to be effective in decreasing TPR in adrenocorticotrophic hormoneinduced hypertensive rats. ${ }^{11}$ Using the same dose and mode of administration, TPR in both saline- and DEX-treated rats was significantly decreased. Despite these changes, oral minoxidil did not significantly decrease tail-cuff SBP, direct MAP or direct DBP of DEX-hypertensive rats. In contrast, the direct SBP of DEX-induced hypertensive rats was decreased by minoxidil when measured using the direct method. The discrepancy observed in SBP measurements obtained using the two methods is most likely due to differences in experimental conditions and techniques. Using the direct method, the rats were anesthetized and mechanically ventilated. Tail-cuff SBP, in contrast, was obtained from restrained conscious animals without the effects of general anesthesia and mechanical ventilation. As shown previously, the tail-cuff system is a reliable method for assessing rat SBP and produces results that are comparable with telemetry. ${ }^{19}$

In this study, hemodynamic parameters were measured under pentobarbitone general anesthesia $\left(60 \mathrm{mg} \mathrm{kg}^{-1}\right.$, i.p.). There are reports documenting the effect of anesthetic agents on hemodynamic measurements. ${ }^{20,21}$ Pentobarbitone $\left(50 \mathrm{mg} \mathrm{kg}^{-1}\right.$, i.p.) has been shown to decrease CI and HR in mice. ${ }^{20}$ Koeppen et al. ${ }^{21}$ have also demonstrated that pentobarbitone significantly reduced renal blood flow. Mechanical ventilation can also affect hemodynamic parameters. A changing ventilatory pattern in rats under choralose general anesthesia from spontaneous breathing to artificial ventilation resulted in a significant decrease in $\mathrm{CO}$ and $\mathrm{SV}$, and a significant increase in MAP, HR and TPR. ${ }^{22}$ In view of these limitations, appropriately matched controls, which also underwent similar experimental and surgical conditions, were used to minimize the confounding effects associated with acute hemodynamic experiments.

Although direct SBP of DEX-hypertensive rats was significantly reduced by minoxidil, increases in direct MAP and DBP due to DEX were not significantly lowered. Furthermore, DBP in the minoxidil+saline group was significantly increased compared with that in the saline-only group. There are two possible explanations for this absence of an antihypertensive effect of minoxidil. First, the increase in TPR is not critical to the development of DEX-HT, although it might contribute to the maintenance of the hypertensive state. Second, the antihypertensive effect of minoxidil may have been offset by volume factors. CO and CI, in both minoxidil+saline- and minoxidil+DEXtreated rats, were increased. As there was no change in HR between treatment groups, minoxidil-induced increases in CO and CI were also associated with increases in SV and SI in the saline and DEX-treated rats. It is likely that these increases counteracted the vasodilator effect of minoxidil. The mechanism responsible for the increases in $\mathrm{CO}$ and $\mathrm{CI}$ and the loss of blood pressure-lowering effect of minoxidil is mainly related to plasma volume expansion. In this study, we observed a decrease in hematocrit due to minoxidil in the saline-treated group. Several studies have also suggested that minoxidil treatment resulted in plasma and blood volume expansion in rats. ${ }^{23-25}$ Minoxidil did not alter hematocrit in DEX-HT rats despite increasing CO and CI. It remains unclear whether DEX can inhibit the decrease in hematocrit due to minoxidil.

In conclusion, DEX-HT is associated with increased TPR. Concomitant treatment with the vasodilator minoxidil prevented the increase in TPR but did not prevent DEX-HT, which was associated with increase in CO and CI. Thus, increased TPR is a feature of DEX-HT that is not critical for the production of DEX-HT.

\section{CONFLICT OF INTEREST}

The authors declare no conflict of interest.

\section{ACKNOWLEDGEMENTS}

Dr SLH Ong was supported by the Jacquot Research Entry Scholarship from the Royal Australasian College of Physicians and a Scholarship (award number: PB07C3427) from the National Heart Foundation of Australia.

1 Ong SLH, Zhang Y, Whitworth JA. Mechanism of dexamethasone-induced hypertension. Curr Hypertens Rev 2009; 5: 61-74

2 Whitworth JA, Gordon D, Andrews J, Scoggins BA. The hypertensive effect of synthetic glucocorticoids in man: role of sodium and volume. J Hypertens 1989; 7: 537-549.

3 Macefield VG, Williamson PM, Wilson LR, Kelly JJ, Gandevia SC, Whitworth JA. Muscle sympathetic vasoconstrictor activity in hydrocortisone-induced hypertension in humans. Blood Press 1998; 7: 215-222. 
4 Wallerath T, Godecke A, Molojavyi A, Li H, Schrader J, Forstermann U. Dexamethasone lacks effect on blood pressure in mice with a disrupted endothelial NO synthase gene. Nitric Oxide 2004; 10: 36-41.

5 Hu L, Zhang Y, Lim PS, Miao Y, Tan C, McKenzie KUS, Schyvens CG, Whitworth JA. Apocynin but not $L$-arginine prevents and reverses dexamethasone-induced hypertension in the rat. Am J Hypertens 2006; 19: 413-418.

6 Pirpiris M, Sudhir K, Yeung S, Jennings G, Whitworth JA. Pressor responsiveness in corticosteroid-induced hypertension in humans. Hypertension 1992; 19: 567-574.

7 Nakamoto H, Suzuki H, Kageyama Y, Ohishi A, Murakami M, Naitoh M, Saruta T. Characterization of alterations of hemodynamics and neuroendocrine hormones in dexamethasone induced hypertension in dogs. Clin Exp Hypertens A 1991; 13: 587-606.

8 Whitworth JA, Schyvens CG, Zhang Y, Mangos GJ, Kelly JJ. Glucocorticoid-induced hypertension: from mouse to man. Clin Exp Pharmacol Physiol 2001; 28: 993-996.

9 Whitworth JA, Zhang Y, Mangos G, Kelly JJ. Species variability in cardiovascular research: the example of adrenocorticotrophin-induced hypertension. Clin Exp Pharmacol Physiol 2006; 33: 887-891.

10 Wen C, Li M, Whitworth JA. Validation of transonic small animal flowmeter for measurement of cardiac output and regional blood flow in the rat. J Cardiovasc Pharmacol 1996; 27: 482-486.

11 Wen C, Fraser T, Li M, Turner SW, Whitworth JA. Haemodynamic mechanisms of corticotropin (ACTH)-induced hypertension in the rat. J Hypertens 1999; 17: 1715-1723.

12 Nakamoto H, Suzuki H, Kageyama Y, Murakami M, Naitoh M, Saruta T. Central nervous system mediates an antihypertensive property in glucocorticoid hypertension in dogs. J Hypertens 1995; 13: 1169-1179.

13 Tonolo G, Fraser R, Connell JM, Kenyon CJ. Chronic low-dose infusions of dexamethasone in rats: effects on blood pressure, body weight and plasma atrial natriuretic peptide. J Hypertens 1988; 6: 25-31.

14 Mangos G, Walker B, Williamson P, Whitworth J, Kelly J. Effect of synthetic corticosteroids on vascular reactivity in the human forearm. Clin Exp Hypertens 2006; 28: 707-718.
15 Wallerath T, Witte K, Schafer SC, Schwarz PM, Prellwitz W, Wohlfart P, Kleinert H, Lehr $\mathrm{H}-\mathrm{A}$, Lemmer B, Forstermann U. Down-regulation of the expression of endothelial NO synthase is likely to contribute to glucocorticoid-mediated hypertension. Proc Natl Acad Sci USA 1999; 96: 13357-13362.

16 Handa M, Kondo K, Suzuki H, Saruta T. Dexamethasone hypertension in rats: role of prostaglandins and pressor sensitivity to norepinephrine. Hypertension 1984; 6: 236-241.

17 Gardiner SM, Kemp PA, March JE, Bennett T. Effects of dexamethasone and SB 209670 on the regional haemodynamic responses to lipopolysaccharide in conscious rats. Br J Pharmacol 1996; 118: 141-149.

18 Chabria NL, Gaitonde BB. Effect of dexamethasone on urine output and electrolyte excretion in rats. Arch Int Pharmacodyn Ther 1966; 162: 364-370.

19 Fraser TB, Turner SW, Mangos GJ, Ludbrook J, Whitworth JA. Comparison of telemetric and tail-cuff blood pressure monitoring in adrenocorticotrophic hormone-treated rats. Clin Exp Pharmacol Physiol 2001; 28: 831-835.

20 Janssen BJA, De Celle T, Debets JJM, Brouns AE, Callahan MF, Smith TL. Effects of anesthetics on systemic hemodynamics in mice. Am J Physiol Heart Circ Physiol 2004; 287: H1618-H1624.

21 Koeppen BM, Katz Al, Lindheimer MD. Effect of general anaesthesia on renal haemodynamics in the rat. Clin Sci (Lond) 1979; 57: 469-471.

22 Sellden H, Sjovall H, Ricksten SE. Sympathetic nerve activity and central haemodynamics during mechanical ventilation with positive end-expiratory pressure in rats. Acta Physiol Scand 1986; 127: 51-60.

23 Leenen FH, Prowse S. Time-course of changes in cardiac hypertrophy and pressor mechanisms in two-kidney, one clip hypertensive rats during treatment with minoxidil, enalapril or after uninephrectomy. J Hypertens 1987; 5: 73-83.

24 Tsoporis J, Fields N, Lee RM, Leenen FH. Arterial vasodilation and cardiovascular structural changes in normotensive rats. Am J Physiol Heart Circ Physiol 1991; 260: H1944-H1952.

25 Sanz E, Lopez Novoa JM, Linares M, Digiuni E, Caramelo CA. Intravascular and interstitial fluid dynamics in rats treated with minoxidil. J Cardiovasc Pharmacol 1990; 15: 485-492. 